\title{
ESPUMA DE MAGNÉSIO: AVALIAÇÃO DA DENSIDADE RELATIVA DE AMOSTRAS OBTIDAS A PARTIR DE PRENSAGEM UNIAXIAL DE MISTURAS DE PÓS DE LIGA DE MAGNÉSIO AZ91 COM HIDRETO DE TITÂNIO*
}

Lucas Xavier Soares de Souza ${ }^{1}$ Cristina Aparecida Esteves ${ }^{2}$ Sidney Nicodemos da Silva ${ }^{3}$ Ivete Peixoto Pinheiro ${ }^{4}$

\section{Resumo}

No contexto de eficiência energética nos setores automobilístico e aeronáutico, a redução de peso em estruturas assume um papel importante. Como alternativa para isso, as espumas metálicas, mais especificamente, as espumas de magnésio e de ligas de magnésio, surgem com grande potencial. Isso se deve, primeiramente, às propriedades desse elemento químico, que é o mais leve entre os metais estruturais. Já a estrutura em forma de espuma aparece como fator capaz de potencializar as propriedades desse elemento. Um dos processos para produção de espumas de magnésio é a metalurgia do pó e uma das etapas desse processo é a compactação dos pós. Busca-se com este trabalho, avaliar a densidade relativa de amostras que foram compactadas a partir da prensagem uniaxial a frio de pós da liga de magnésio AZ91 com hidreto de titânio. Foi verificado que para uma mesma pressão, variando a granulometria da liga AZ91 e a quantidade de hidreto, a variação nas densidades relativas é desprezível. A partir das curvas obtidas nos ensaios de compactação foi possível verificar que na faixa de 130 a $230 \mathrm{MPa}$ ocorreu deformação plástica dos pós de forma gradual e foi possível constatar que a partir de $300 \mathrm{MPa}$ não ocorre variação significativa da densidade relativa com o aumento da pressão.

Palavras-chave: Magnésio; Espuma de magnésio; Metalurgia do pó; Prensagem uniaxial.

\section{MAGNESIUM FOAM: EVALUATION OF RELATIVE DENSITY OF COMPACT SPECIMENS OBTAINED FROM UNIAXIAL PRESSURE OF AZ91 MAGNESIUM ALLOY POWDERS MIXED WITH TITANIUM HYDRIDE}

\section{Abstract}

In the context of energy efficiency in the automotive and aeronautical sectors, weight reduction in structures plays an important role. As an alternative to this, metallic foams, more specifically, magnesium and magnesium alloy foams, come with great potential. This is due, first, to the properties of this chemical element, which is the lightest among the structural metals. The foam structure appears as a factor capable of enhancing the properties of this element. One of the processes for the production of magnesium foams is powder metallurgy and one of the steps in this process is the compacting of the powders. The aim of this work was to evaluate the relative density of samples that were compacted from the uniaxial cold pressing of powders of magnesium alloy AZ91 with titanium hydride. It has been found that for the same pressure, varying the granulometry of alloy AZ91 and the amount of hydride, variation in relative densities is negligible. From the curves obtained in the compaction tests it was possible to verify that in the range of 130 to $230 \mathrm{MPa}$ plastic deformation of the powders occurred gradually and it was possible to verify that from $300 \mathrm{MPa}$ there is no significant variation of the relative density with the increase of the pressure.

Keywords: Magnesium; Magnesium foam; Powder metallurgy; Uniaxial pressing. 
1 Engenheiro Industrial Mecânico, Departamento de Engenharia de Materiais, Centro Federal de Educação Tecnológica de Minas Gerais, Belo Horizonte, MG, Brasil.

2 Engenheira de Materiais, Departamento de Engenharia de Materiais, Centro Federal de Educação Tecnológica de Minas Gerais, Belo Horizonte, MG, Brasil.

3 Físico, Doutor, Professor, Departamento de Engenharia de Materiais, Centro Federal de Educação Tecnológica de Minas Gerais, Belo Horizonte, MG, Brasil.

4 Engenheira Metalurgista, Doutora, Professora titular, Departamento de Engenharia de Materiais, Centro Federal de Educação Tecnológica de Minas Gerais, Belo Horizonte, MG, Brasil. 


\section{INTRODUÇÃO}

Devido à tendência de se reduzir ao máximo o consumo de combustível no transporte, a utilização de magnésio surge como uma alternativa para as indústrias automotiva e aeronáutica, para reduzir peso e, consequentemente diminuir o consumo de combustível. Isso se deve ao fato do magnésio ser o metal estrutural com menor densidade: $1,74 \mathrm{~g} / \mathrm{cm}^{3}$ [1]. Embora as ligas de magnésio apresentem resistência ao escoamento inferior às de outras ligas, como ligas de alumínio, de titânio, aços inoxidáveis austeníticos e aços de alta resistência mecânica e baixa liga, a razão resistência/peso do magnésio é comparável às das ligas de alumínio e de titânio, e muito superior às dos dois tipos de aços citados [2]. Além disso, ao combinar as propriedades do magnésio com a estrutura em forma de espuma, essas podem ser potencializadas. Além de benefícios na redução de massa, as espumas de magnésio podem contribuir para uma maior segurança passiva das pessoas em caso de colisões com automóveis por exemplo, pois esses materiais tendem a ter alta eficiência em absorção de energia em compressão, reduzindo, assim, os efeitos do impacto no corpo humano.

O objetivo deste trabalho é avaliar as densidades relativas das amostras que foram obtidas a partir da prensagem uniaxial dos pós de liga de magnésio em granulometrias distintas e misturados com agente expansor em diferentes percentuais em peso.

A densidade relativa é determinada relacionando a densidade medida e a densidade teórica da liga utilizada [3].

A compactação dos pós é uma das etapas do processo de metalurgia do pó. Essa compactação serve para romper as camadas de óxido na superfície dos pós, com a finalidade de estabelecer uma ligação metalúrgica inicial, e aprisionar os pós de agente expansor [3]. A pressão de compactação deve ser suficiente para quebrar as camadas de óxido na superfície do magnésio, mas não tão grande que deforme as partículas do agente expansor [4]. A pressão a ser utilizada para compactação das amostras será definida com base nas propriedades da liga, nas curvas de compactação densidade relativa versus pressão de compactação e em trabalhos anteriores. No presente trabalho, a compactação dos pós será feita por prensagem uniaxial a frio (temperatura ambiente). Posteriormente, esses corpos de prova serão levados ao forno para expandir e formar a estrutura em forma de espuma. As espumas metálicas resultantes deste processo têm uma estrutura porosa fechada e uma superfície densa que melhora as propriedades mecânicas destes materiais [5].

Essa expansão é devida à decomposição do agente expansor. Qualquer material sólido pode ser utilizado como agente expansor, porém, na prática, a seleção do agente expansor é limitada porque este material deve ser removido ou agregado de forma que não prejudique as características do produto final [4]. Os agentes expansores geralmente utilizados no processo de metalurgia do pó são os hidretos metálicos que são compostos formados entre o hidrogênio e metais de transição [5]. Para o presente trabalho, o agente expansor utilizado será o hidreto de titânio.

As propriedades mecânicas das espumas de magnésio podem ser alteradas a partir da variação do tamanho e da distribuição dos poros que, por sua vez, dependem do controle do tamanho e da forma dos pós e das condições de processo como pressão de compactação, tempo e temperatura de sinterização [6]. 


\section{MATERIAIS E MÉTODOS}

\subsection{Materiais}

Para o presente trabalho foi utilizado pós da liga de magnésio AZ91 fornecido pela empresa RIMA INDUSTRIAL S/A e pós de hidreto de titânio $\left(\mathrm{TiH}_{2}\right)$ como agente expansor. A tabela 1 apresenta composição química da liga de acordo com 0 fornecedor e a tabela 2 apresenta a distribuição granulométrica do agente expansor que foi obtida por meio de um granulômetro a laser modelo 1190 da marca CILAS. Os pós da liga foram definidos como AZ91-tipo 1 para faixa granulométrica de 100-

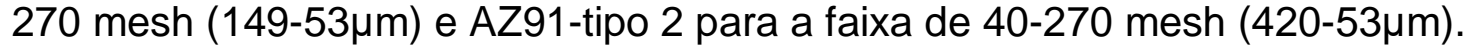

Tabela 1. Composição química da liga AZ91.

\begin{tabular}{ccccccccc}
\hline$\% \mathrm{Mg}$ & $\% \mathrm{Al}$ & $\% \mathrm{Zn}$ & $\% \mathrm{Mn}$ & $\% \mathrm{Cu}$ & $\% \mathrm{Si}$ & $\% \mathrm{Fe}$ & $\% \mathrm{Ni}$ & $\%$ Outros \\
\hline 90,352 & 8,8 & 0,56 & 0,23 & 0,005 & 0,04 & 0,002 & 0,001 & 0,01 \\
\hline Composição química de acordo com certificado & da empresa & RIMA INDUSTRIAL S/A. &
\end{tabular}

Tabela 2. Distribuição granulométrica dos pós de hidreto de titânio $\left(\mathrm{TiH}_{2}\right)$. Hidreto de Titânio $\quad$ D 90, $\mu \mathrm{m} \quad$ D 50, $\mu \mathrm{m} \quad \mathrm{D} 10, \mu \mathrm{m}$ Diâmetro médio, $\mu \mathrm{m}$ $\begin{array}{lllll}\mathrm{TiH}_{2} & 31,61 & 16,61 & 5,22 & 17,76\end{array}$

Os aspectos geométricos dos pós da liga AZ91 são apresentados na figura 1. Essas figuras foram obtidas em um microscópio eletrônico de varredura (MEV), modelo VEGA3 TESCAN. Pode ser verificado que as partículas apresentam geometrias variadas com tendência flocular sem a presença de quinas.
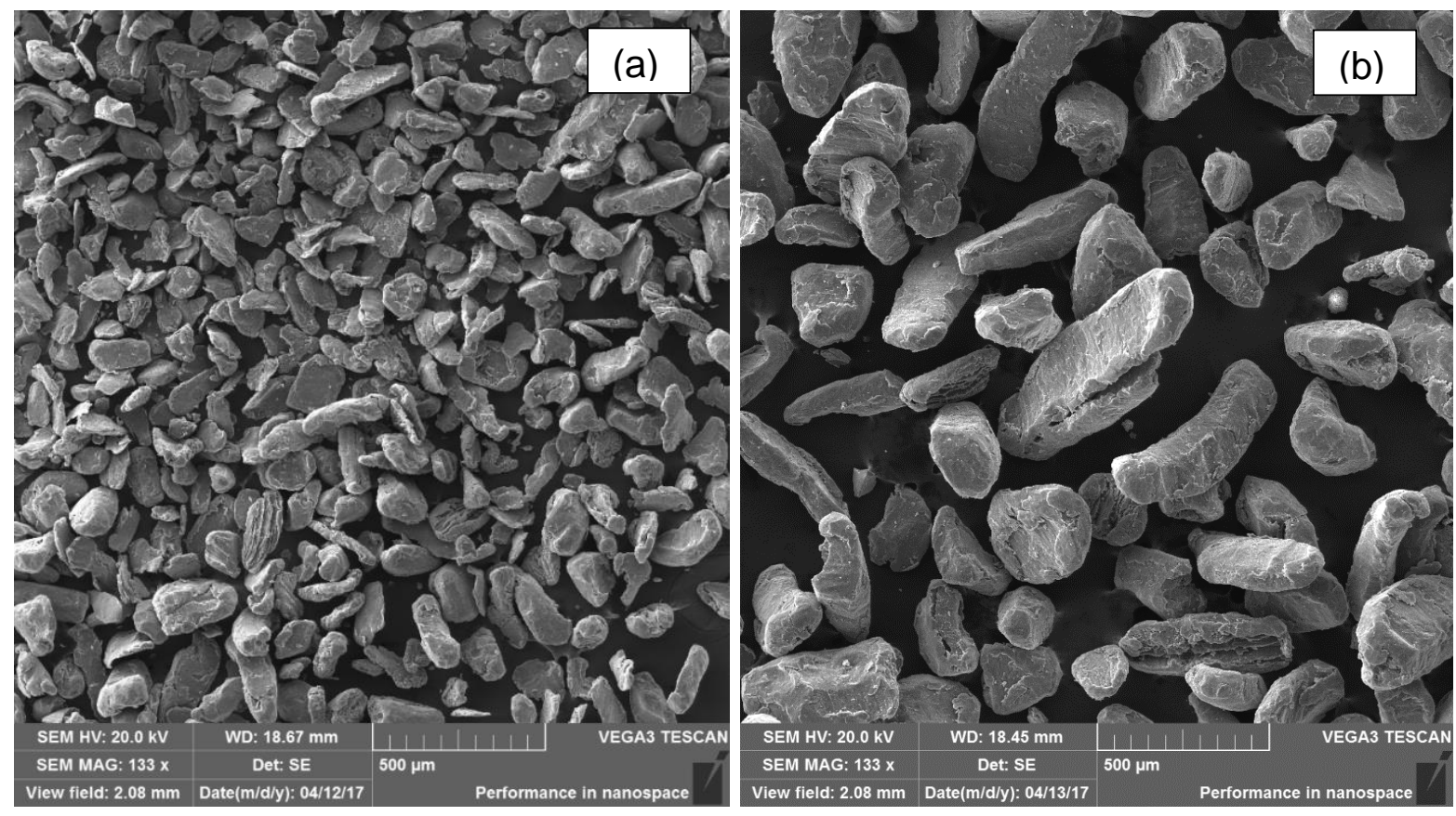

Figura 1. Aspecto geométrico dos pós, (a) AZ91-tipo 1 e (b) AZ91-tipo 2. 


\subsection{Metodologia}

As amostras foram obtidas a partir de prensagem uniaxial de misturas de pós da liga de magnésio com hidreto de titânio. Foram utilizados duas granulometrias diferentes de liga de magnésio e duas porcentagens em peso de hidreto de titânio $(0,3$ e $0,6 \%)$. Esses pós foram misturados em um misturador manual em $Y$ para alcançar a maior homogeneidade possível. Após a mistura, os pós foram inseridos em uma matriz com diâmetro de $25 \mathrm{~mm}$ (figura 2). Os pós foram adequadamente separados para gerar amostras na faixa de 20 a 25 gramas. Para a prensagem uniaxial foi utilizado uma prensa hidráulica modelo SL12 do fabricante SOLAB CIENTÍFICA com capacidade de 20 toneladas (figura 3).

Inicialmente foram realizados ensaios com o intuito de gerar as curvas de compactação, para estas auxiliarem na definição da melhor pressão de compactação, P. Para isso foi utilizada uma máquina universal de ensaios modelo EMIC 23-300, eletromecânica, microprocessada, marca INSTRON/EMIC com capacidade de $300 \mathrm{kN}$ (figura 4). Foram realizados 4 ensaios de acordo com os parâmetros da tabela 3 .

Tabela 3. Parâmetros para ensaios de compactação.

\begin{tabular}{ccccccc}
\hline Ensaios & Liga & $\begin{array}{c}\text { \% em peso } \\
\text { de agente } \\
\text { expansor }\end{array}$ & $\begin{array}{c}\text { Quantidade } \\
\text { de mistura } \\
(\mathrm{g})\end{array}$ & Temperatura & $\begin{array}{c}\text { Pressão } \\
\text { máxima } \\
(\mathrm{MPa})\end{array}$ & $\begin{array}{c}\text { Velocidade da } \\
\text { prensagem } \\
\text { uniaxial }\end{array}$ \\
\hline E1 & AZ91-tipo 2 & 0,6 & Aprox. 25 & $18{ }^{\circ} \mathrm{C}$ & 530 & $2 \mathrm{~mm} / \mathrm{min}$ \\
\hline E2 & AZ91-tipo 1 & 0,6 & Aprox. 25 & $18{ }^{\circ} \mathrm{C}$ & 530 & $2 \mathrm{~mm} / \mathrm{min}$ \\
\hline E3 & AZ91-tipo 1 & 0,3 & Aprox. 25 & $18{ }^{\circ} \mathrm{C}$ & 530 & $4 \mathrm{~mm} / \mathrm{min}$ \\
\hline E4 & AZ91-tipo 2 & 0,3 & Aprox. 25 & $18{ }^{\circ} \mathrm{C}$ & 530 & $4 \mathrm{~mm} / \mathrm{min}$ \\
\hline
\end{tabular}

Para a produção das amostras, a fim de se obter a pressão $P$ definida, 0 processo de prensagem foi dividido em etapas, em um tempo total de 180 segundos de acordo com o procedimento da tabela 4.

Tabela 4. Procedimento de prensagem.

\begin{tabular}{ll}
\hline Etapa 1 & 30 s com pressão de $20 \%$ de $\mathrm{P}$ \\
\hline Etapa 2 & 30 s com pressão de $40 \%$ de $\mathrm{P}$ \\
\hline Etapa 3 & 30 s com pressão de $60 \%$ de $\mathrm{P}$ \\
\hline Etapa 4 & 30 s com pressão de $80 \%$ de $\mathrm{P}$ \\
\hline Etapa 5 & 60 s com pressão de $100 \%$ de $\mathrm{P}$ \\
\hline
\end{tabular}




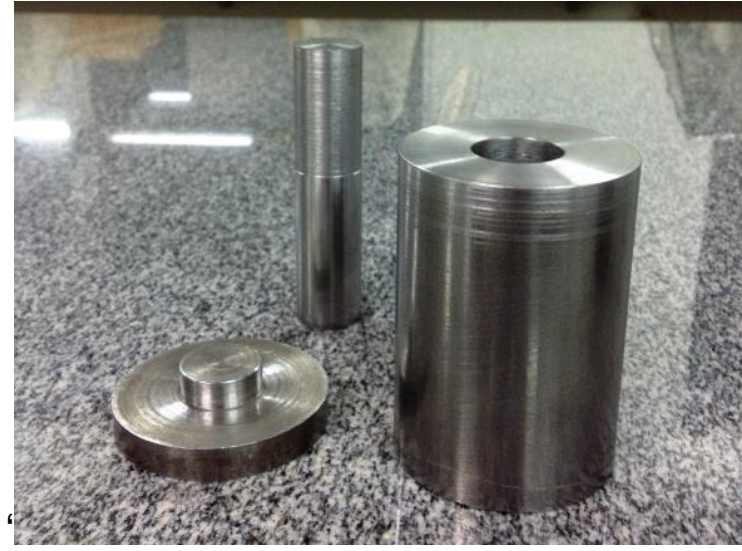

Figura 2. Matriz utilizada na prensagem uniaxial.

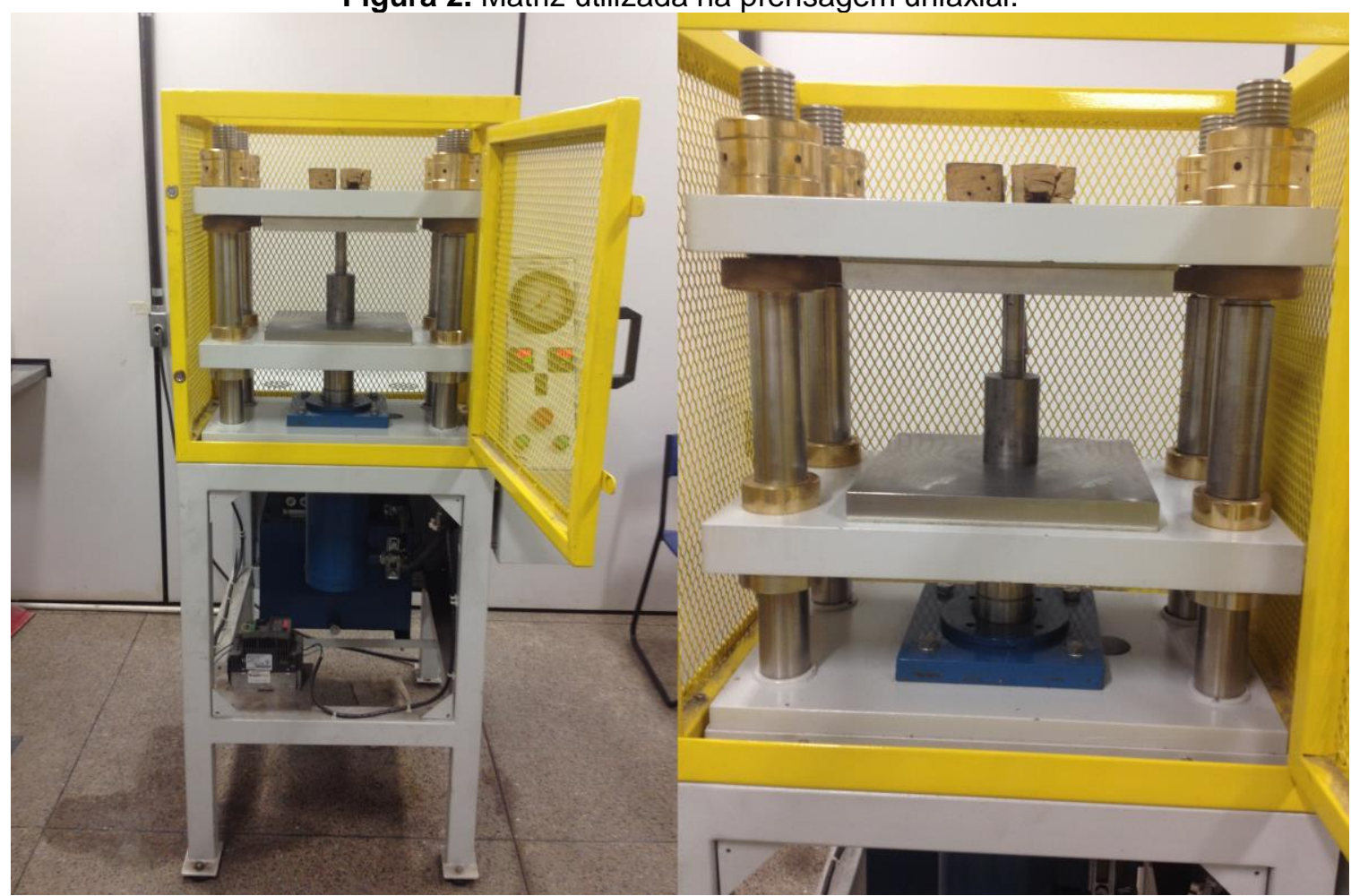

Figura 3. Prensa e matriz utilizada na prensagem uniaxial. 


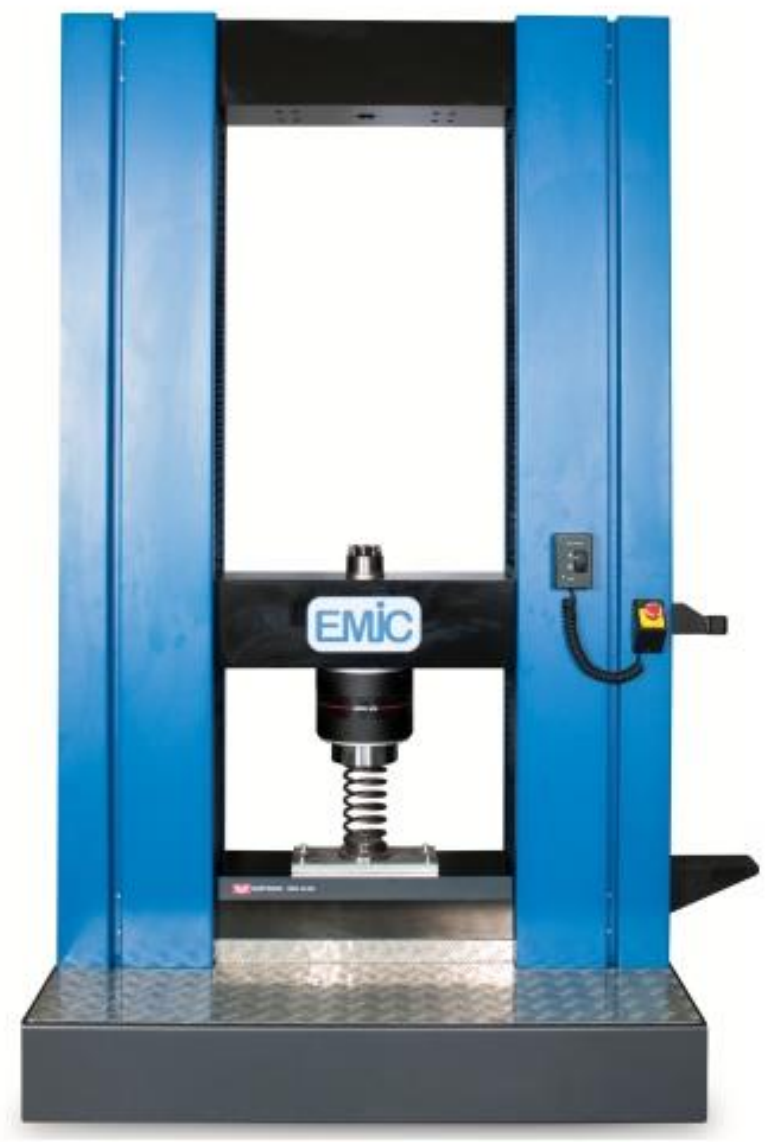

Figura 4. Máquina universal de ensaios, modelo EMIC 23-300, marca INSTRON/EMIC.

Para avaliação de densidade das amostras produzidas, foram utilizados dois métodos. O primeiro método utilizado foi baseado no princípio de Arquimedes e o meio utilizado foi água destilada. Para este método, com a finalidade de não danificar a amostra devido a absorção de água, utilizou-se filme plástico de PVC recobrindo cada amostra. O segundo, chamado método geométrico, consistiu em verificar a dimensão das amostras. Essa verificação foi feita utilizando um micrômetro de $0-25 \mathrm{~mm}$ com graduação de $0,01 \mathrm{~mm}$. Foram realizadas medidas em três pontos da altura e três pontos do diâmetro, conforme figura 5; para o cálculo do volume foi utilizado a média aritmética simples do diâmetro e da altura. A análise da densidade pelo método geométrico também foi feita com a amostra recoberta com filme plástico. Para os dois métodos utilizou-se uma balança eletrônica de precisão com graduação de $0,0001 \mathrm{~g}$. Para fins de resultado serão descartados os maiores e menores valores de cada conjunto de 4 amostras com a finalidade de diminuir 0 impacto de erros. 


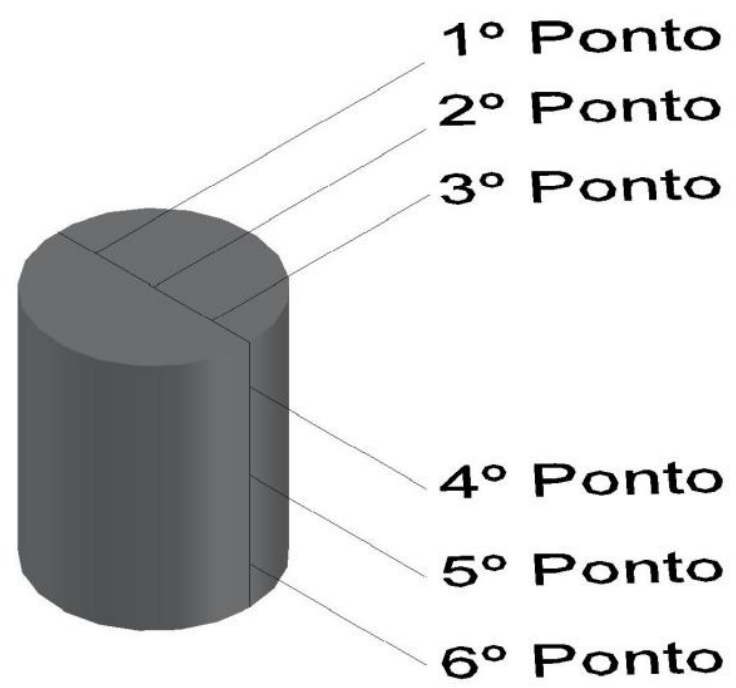

Figura 5. Metodologia de medição para método geométrico.

\section{RESULTADOS E DISCUSSÃO}

Foram produzidas um total de 16 amostras, sendo 8 para cada tipo de pó da liga. Dessas 8 amostras, 4 foram feitas utilizando $0,3 \%$ em peso de $\mathrm{TiH}_{2}$ e as outras 4 restantes com $0,6 \%$ em peso do mesmo hidreto. Exemplos das amostras produzidas estão representadas na figura 6.

As curvas de compactação estão apresentadas na figura 7. Nesse gráfico pode-se verificar que na faixa de 130 a $230 \mathrm{MPa}$, as curvas apresentam uma forma serrilhada. A explicação para isso pode ser o fato da liga AZ91 apresentar limite de escoamento compressivo, em $0,2 \%$, de $165 \mathrm{MPa}$ [9]. Como na prensagem uniaxial a distribuição de pressão não é homogênea, a faixa serrilhada provavelmente se dá porque, em um primeiro momento, alguns pós atingem a pressão de escoamento e, de forma gradativa os outros pós também deformam, até que depois de uma certa pressão aplicada todo material escoa.

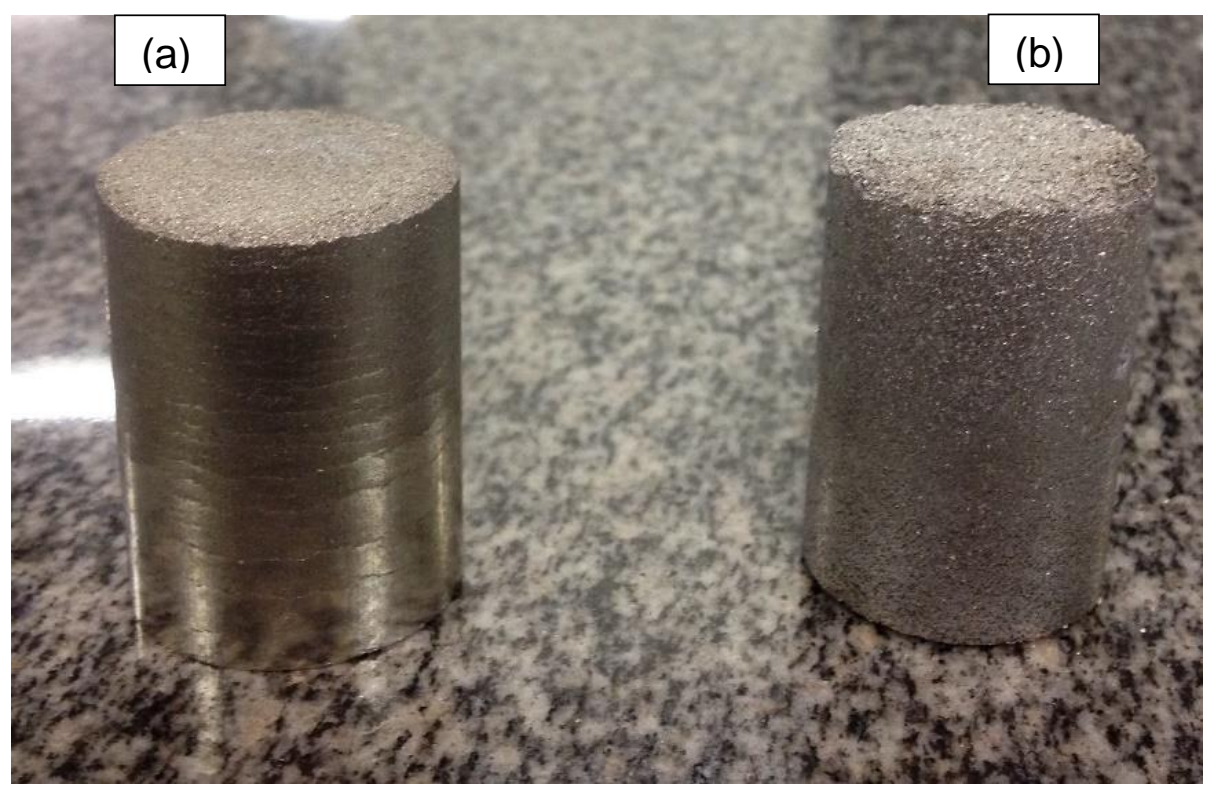


Figura 6. Exemplos de amostras produzidas: (a) amostra AZ91-tipo 1 e (b) amostra AZ91-tipo 2.

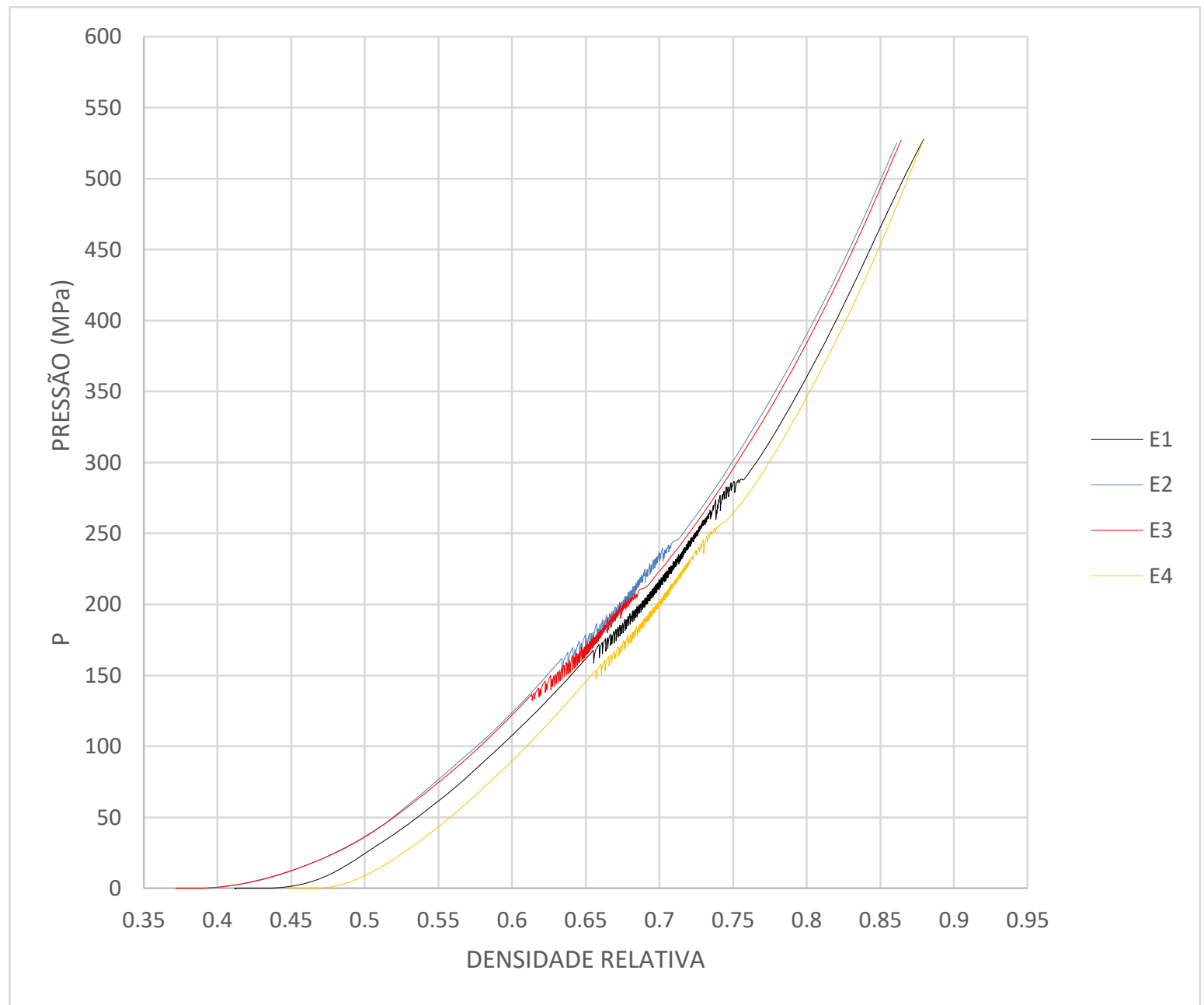

Figura 7. Curvas de compactação.

A partir das curvas de compactação e de resultados de trabalhos anteriores $[3,5,7]$ pode-se definir a melhor pressão como $300 \mathrm{MPa}$. Como pode ser visto no gráfico da figura 7 , em $300 \mathrm{MPa}$ os pós já deformaram plasticamente e, provavelmente, aprisionaram os pós de agente expansor. Além disso, em baixas pressões de compactação $(<150 \mathrm{MPa})$ o filme de óxido na superfície dos pós pode não ser rompido, o que pode ser prejudicial no processo de expansão da espuma [7] e em pressões de compactação superiores a 300MPa, apesar de gerar ótima ligação entres os pós do metal base, na prática é uma pressão difícil de se obter e pode gerar espumas com poros deformados [5, 7].

Os resultados obtidos para as densidades relativas para amostras estão representados na figura 8 . 


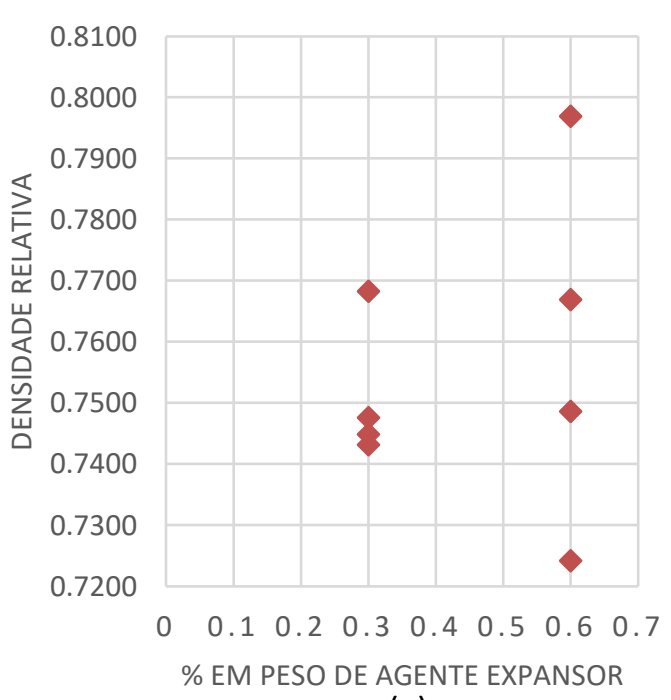

(a)

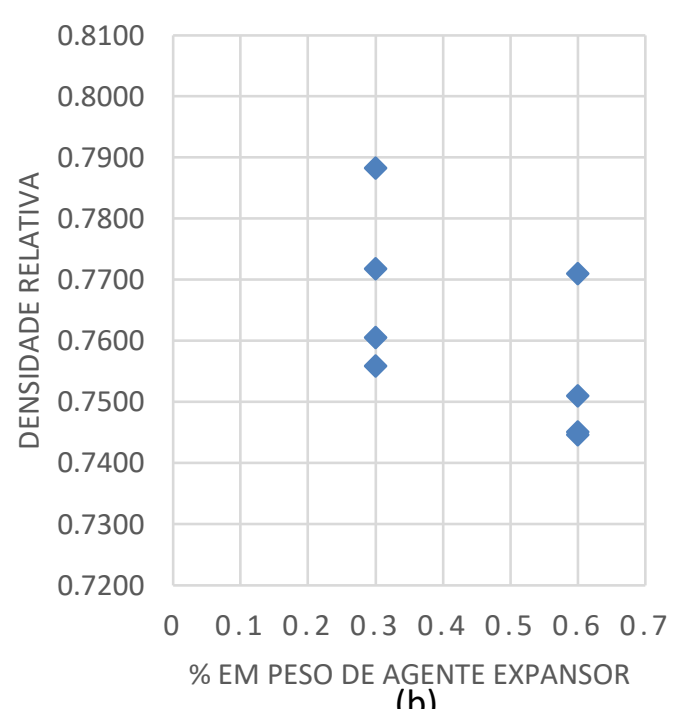

(b)

Figura 8. Resultados de densidade relativa para: (a) AZ91-tipo 1 e (b) AZ91-tipo 2.

Não houve significante variação nas densidades relativa tanto antes quanto após o descarte das amostras como foi citado na metodologia. A densidade relativa calculada foi a relação entre a densidade das amostras e a densidade teórica da liga que é de $1,81 \mathrm{~g} / \mathrm{cm}^{3}$. Considerando todas as amostras, a maior variação ficou em torno de $9 \%$ enquanto que após o descarte a variação não ultrapassou $4 \%$. Considerando o descarte e amostras de um só tipo de granulometria, foi verificado variação de densidade relativa para os pós de AZ91-tipo 1 e AZ91-tipo 2 ficaram em torno de $3 \%$ e $3,5 \%$ respectivamente.

Esses resultados indicam que a pressão utilizada na prensagem uniaxial está sendo suficientemente alta para garantir a compactação dos pós de liga de magnésio, pois mesmo com a variação na granulometria, os pós foram deformados de forma a ficarem com densidades relativas semelhantes.

Não foi possível verificar a influência do hidreto de titânio na densidade relativa das amostras produzidas. Isso provavelmente se deve ao fato da pequena quantidade utilizada desse material bem como do seu tamanho, que é bem menor do que o tamanho das partículas de liga AZ91.

A densidade relativa das amostras variou de 74 a $77 \%$, resultado que está em discordância com resultados de outros autores que obtiveram para a mesma pressão valores em torno de 92\% para o alumínio [8] e 99\% para magnésio [5], entretanto, o último utilizou prensagem uniaxial a quente com $400^{\circ} \mathrm{C}$.

\section{CONCLUSÃO}

Por meio das curvas de compactação (figura 7), pôde-se avaliar o comportamento dos pós no decorrer do tempo em que a carga é aplicada e constatar que a pressão de $300 \mathrm{MPa}$, utilizada em trabalhos prévios de outros autores, possibilita a deformação plástica na compressão de todo o pó no caso das amostras do presente trabalho. Nesse gráfico também pode ser visto que a partir de $300 \mathrm{MPa}$ a variação da densidade com o aumento da pressão é pequena o que é um indicativo de pouca movimentação entre os pós, ou seja, poucos espaços vazios.

Além das informações das curvas de compactação, foram produzidas amostras compactadas por prensagem uniaxial a frio de pós da liga de magnésio AZ91. Os resultados mostraram que a granulometria e a variação da quantidade de 
agente expansor não impactaram na densidade relativa das amostras geradas utilizando-se pressão de $300 \mathrm{MPa}$. É importante ressaltar que apesar de densidades semelhantes, as amostras do AZ91-tipo 2 apresentam esboroamento em uma das faces do cilindro (ver figura 6), e isso pode ser explicado pela diferença de densidade no corpo da amostra. Essa diferença de densidade é característica da prensagem uniaxial, pois devido ao atrito entre os pós e entre estes e a parede da matriz, um gradiente de pressão é gerado e isso pode ser amenizado reduzindo a razão entre altura e diâmetro da amostra. Além disso, na figura 6 também pode ser observada a formação de fissuras (paralelas às faces do cilindro) que pode ser um problema na fase de expansão da espuma, pois os gases gerados na decomposição do hidreto podem escapar da amostra e, dessa forma, prejudicar os resultados de densidade da espuma.

\section{Agradecimentos}

Os autores agradecem à FAPEMIG e à FINEP pelo apoio financeiro, ao Departamento de Engenharia de Materiais do CEFET-MG pela infraestrutura oferecida, e à empresa RIMA INDUSTRIAL S/A pela doação dos pós de liga de magnésio.

\section{REFERÊNCIAS}

1 Callister WD, Rethwisch DG. Materials science and engineering: an introduction. 8. ed. Danvers: John Wiley \& Sons, 2009.

2 Barbosa C. Metais não ferrosos e suas ligas: microestrutura, propriedades e aplicações. Rio de Janeiro: E-papers, 2014.

3 Duarte IMA. Espumas metálicas: processo de fabricação, caracterização e simulação numérica [dissertação de mestrado]. Porto: Faculdade de Engenharia da Universidade do Porto; 2005.

4 Čapek J, Vojtěch D. Effect of sintering conditions on the microstructural and mechanical characteristics of porous magnesium materials prepared by powder metallurgy. Materials science and engineering C. 2013; 35: 21-28.

$5 \mathrm{Neu}$ TR et al. Magnesium and magnesium alloy foams. In: International conference on porous metals and metallic foams. Proceedings 7. Metfoam; 2011; Busan, Korea: 2011. p. 133-140.

6 Čapek J, Vojtěch D. Properties of porous magnesium prepared by powder metallurgy. Materials Science and Engineering C.2012; 33: 564-569.

7 Hao GL, Han FS. Li WD. Processing and mechanical properties of magnesium foams. J Porous Mater. 2008; 16: 251-256.

8 Bonaldi PO. Obtenção de espuma de alumínio através do processo de metalurgia do pó e propriedades mecânicas de estrutura sanduíche [tese de doutorado]. Porto Alegre: Universidade Federal do Rio Grande do Sul; 2012.

9 Avedesian MM, Baker H. ASM Specialty Handbook: Magnesium and Magnesium Alloys. Ohio: Materials Park. ASM International, 1999 